Literatur nach der Digitalisierung 
Gegenwartsliteratur -
Autoren und Debatten 


\section{Literatur nach der Digitalisierung}

Zeitkonzepte und Gegenwartsdiagnosen

Herausgegeben von

Elias Kreuzmair und Eckhard Schumacher 
Der Sammelband ist im Rahmen des Forschungsprojekts „Schreibweisen der Gegenwart.

Zeitreflexion und literarische Verfahren nach der Digitalisierung“ entstanden, gefördert durch die Deutsche Forschungsgemeinschaft (DFG) - Projektnummer 426792415.

Die Open-Access-Publikation wurde durch die Universität Greifswald gefördert.

ISBN 978-3-11-075843-6

e-ISBN (PDF) 978-3-11-075860-3

e-ISBN (EPUB) 978-3-11-075866-5

ISSN 2567-1219

DOI https://doi.org/10.1515/9783110758603

\section{(c) BY-NC-ND}

Dieses Werk ist lizenziert unter einer Creative Commons Namensnennung - Nicht-kommerziell Keine Bearbeitung 4.0 International Lizenz. Weitere Informationen finden Sie unter http://creativecommons.org/licenses/by-nc-nd/4.0/.

\section{Library of Congress Control Number: 2021944008}

\section{Bibliografische Information der Deutschen Nationalbibliothek}

Die Deutsche Nationalbibliothek verzeichnet diese Publikation in der Deutschen Nationalbibliografie; detaillierte bibliografische Daten sind im Internet über http://dnb.dnb.de abrufbar.

(C) 2022 bei den Autoren und Autorinnen, Zusammenstellung @ 2022 Elias Kreuzmair, Eckhard Schumacher, publiziert von Walter de Gruyter GmbH, Berlin/Boston Dieses Buch ist als Open-Access-Publikation verfügbar über www.degruyter.com.

Umschlagfotos: (c) Magdalena Pflock

Satz: Integra Software Services Pvt. Ltd.

Druck und Bindung: CPI books GmbH, Leck

www.degruyter.com 